\title{
Internet der Dinge - quo vadis?
}

\section{Das Industrielle Internet als Chance für den europäischen Industriestandort}

\author{
G. Kopetz \\ Online publiziert am 11. November 2016 \\ (C) Springer Verlag Wien 2016
}

Wir wissen heute, dass die Entwicklung des Internets mit der digitalen Kommunikation zwischen Menschen begonnen hat und in den letzten Jahren mit zunehmender Verbreitung von Videostreaming und dem "social web" eine Hochblüte in Verbindung mit unseren mobilen Kommunikationsgeräten erlebt. Die europäische Technologiebranche spielt in diesen ersten Kapiteln der Geschichte des Internets nur eine wenig beachtete Nebenrolle.

In einem anderen Bereich der Computerindustrie, der industriellen Steuerungs- und Vernetzungstechnik, zum Beispiel im Maschinenbau und in der Fahrzeugtechnik, sind europäische Unternehmen hingegen global führend. Europäer haben wiederholt international Standards gesetzt und sind durch ihre intelligenten "cyber-physischen" Lösungen Weltmarktführer in vielen Branchen. Als fast schon historisches Beispiel sei hier der vor ca. 25 Jahren von Bosch entwickelte CAN-Bus erwähnt, der heute in Milliarden Stückzahlen in Maschinen und Fahrzeugen auf allen Kontinenten verbaut ist.

Bereits seit einigen Jahren sehen wir nunmehr einen stetigen Anstieg der Internet-basierten Maschinenkommunikation (M2M) und eine rasch wachsende Vernetzung von Milliarden von intelligenten ( „smart") Sensoren und Aktuatoren im Industrial Internet of Things (IloT). lloT-Systeme können künftig verstärkt die technische Basis des neuen IEEE TSN Real-Time Ethernet-Standards und die Entwicklungen der OPC Foundation, die eine durchgehende, sichere und offene Kommunikationsarchitektur vom Sensor zur Cloud im Fokus hat, nutzen. IloT-basierte Computersysteme werden dann weitgehend autonom - d. h. unabhängig von menschlicher computergestützten Intervention - bisher klassische industrielle (Geschäfts-)Prozesse steuern und kontinuierlich (auch mit Hilfe von unterschiedlichsten Cloud-Technologien) optimieren. Eine neue Ära der Automatisierung (Industrie 4.0.) hat begonnen: das Zeitalter der Netzwerk- basierten Digitalisierung. Dieser Trend zur IloT-basierten Steuerung wird zudem durch die zahlreichen Sensor- und Softwareinnovationen für das automatisierte Fahren weiter beschleunigt.

Auch die interessierte Fachöffentlichkeit ist von der Geschwindigkeit dieser zunehmenden Verschmelzung der klassischen Internettechnologien mit den Echtzeit- und sicherheitsorientierten Steuerungstechnologien („operations technology“) immer wieder überrascht. Leider wird der öffentliche Diskurs über die beträchtlichen unternehmerischen Chancen oft durch die (berechtigte) Sorge der Datensicherheit überschattet und nicht ausführlich und breit genug geführt. Hier entsteht nämlich gerade ein neuer Milliardenmarkt.

Gerade europäische Unternehmen können mit dem raschen Einsatz von IloT-Technologien wie dem Echtzeit-Fog oder Edge Cloud Computing in Verbindung mit dem neuen IEEE TSN EthernetEchtzeitstandard interessante Wertschöpfungspositionen im Maschinenbau, in der Fahrzeugtechnik oder in der Energieversorgung erobern und/oder ihre führende Marktstellung verteidigen. So beteiligt sich unser Unternehmen TTTech nicht nur aktiv in den internationalen Standardisierungsgruppen (IEEE, OPC, Open Fog etc.), sondern arbeitet auch mit einer Reihe von weltweit führenden Industrieautomatisierungsunternehmen, wie auch der österreichischen B\&R, erfolgreich im amerikanischen Industrial Internet-Konsortium zUsammen, um die Interoperabilität von verschiedenen IloT-basierten Steuerungs- und Kommunikationssystemen für den Produktionsbereich im Echtbetrieb zu testen.

Ich freue mich über das Zustandekommen dieser e\&i-Ausgabe zum Themenschwerpunkt „Internet der Dinge“. Mögen die folgenden Beiträge Ihnen, liebe Leserinnen und Leser, die bereits vorhandene (industrielle) Potenz beim Internet der Dinge in Österreich aufzeigen und die Basis zur weiteren sachlichen Diskussion legen.
Kopetz, Georg, Vorstand und Mitgründer der TTTech Computertechnik AG, Schönbrunner Straße 7, 1040 Wien, Österreich (E-Mail: georg.kopetz@tttech.com) 